\title{
Defining ecological regionalism - a theoretical perspective
}

\author{
M. A. Yadav \\ School of Architecture and Planning, New Delhi, India
}

\begin{abstract}
This paper is a part of an on-going research which attempts to define ecological regionalism with reference to architecture. The paper firstly describes the problem and the need for ecological regionalism. The definitions of architecture, ecology, region, regionalism are studied and then have tried to define ecological regionalism. The interface between architecture and ecology is explained. Objectives of regionalism are elaborated with suitable examples. 'Regionalism in architecture' is a concept of architectural design based on determinants such as culture climate and resources of the particular place. When these regional contents incorporated appropriately, provide the architecture of a region. In ancient civilizations, architecture was very much regional in character because it evolved purely in response to these regional determinants. The history of civilization shows that man developed through trial and error a fine tradition of regional architecture, which still persists in rural habitats of the world. In the urban centers, our concepts and lifestyles have undergone considerable changes like major technological, cultural transformations, which gave rise to different patterns of building and community city planning. Cities are planned with regard to economic growth, social needs and neighbourhood concerns. Nature is typically perceived as separate from the city. Growing human population figures and resource use are degrading the biosphere, which may be disastrous. Architecture must now take itself seriously as a major consumer of resources and a major user of the world's supplies. Architecture is shaped by the ecology of the place; Ecological regionalism helps to conserve natural and regional identity in architecture.
\end{abstract}

Keywords: architecture, ecology, region, eco-region, regionalism, ecological regionalism. 


\section{Introduction}

In Ancient civilizations architecture was without architects, it evolved over time on trial and error basis, usually referred to as 'Vernacular Architecture'. Architecture has traditionally reflected the unique culture, climate, topography of different regions, as well as the aesthetic preferences of local inhabitants. Over the last fifty years, these regional differences have been steadily disappearing. With the change in life style, technological innovations and socio-economic structure, architecture has changed through the ages and we have come a long way from cave dwellings to skyscrapers. Industrial revolution led to urbanization and enabled high-rise high-density living. The man-made environments have changed as result of continued and extensive urbanization as land use, from beings the contained system to the containing one. (Chermayeff and Tzonis, 1971) In effect, the ecosystems in the biosphere are becoming increasingly saturated with man-made systems. This process of saturation has the overall effect of reducing regionally and globally the self-regulative and assimilative ability of the ecosystems. The overall effect is that people and their built systems have become not less dependent upon the functioning of the ecosystems within the biosphere, but on the contrary, more dependent. With the present built environment, people have created a situation where they must now return to the natural ecological controls, develop new ones. Architecture and ecology have strong interface with each other. "The greatest responsibility of the planner and the architect ... is the protection and development of our habitat. Man has evolved a mutual relationship with nature on earth, but his power to change its surface has grown so tremendously that this may become a curse instead of a blessing." (Walter Gropius, 1956)

This paper looks in to the following questions:

- How to understand architecture, ecology and interface between them.

- What is Region and regionalism in architecture?

- Why regional architecture is important?

- How to define ecological regionalism with reference to architecture?

\section{Definitions}

\subsection{Architecture}

Architecture has interactive relationship with nature and derives its expressions from nature. It is built from materials that have been removed from the natural environment. As a built work also, it modifies the natural environment in terms of microclimate, erosion, deposition, and in terms of surrounding life forms.

Ken Yeang defines architectural design as a form of energy and materials management, where the earth's energy and material resources are managed and assembled by the designer into a temporary form (viz., for the period of use), and then demolished at the end of the period of use, with the materials either recycled within the built environment or assimilated into the natural environment. 
Architecture of a place is based on ecology of a place. Ecological determinants shape the architecture.

\subsection{Ecology}

The word ecology had originally come from the Greek word OIKOLOGOUS; meaning, "everything is related to everything" and also containing the philosophy nothing less out of which everything had come about. This in Indian tradition, in Vedas is described as COSMOS.

"The non-existent was not then,

Nor was the existent,

The earth was not, nor the filament,

Nor that which is beyond."

(Rig. 10.129.1-Nasadiya Sukta)

OIKOLOGIA; the meaning of which is the symbiotic relationship within and between man and his living surrounding environment from which the term ecology had come. Literally, it means the logos, the reflection on or studies of the oikos, or household. Ecology thus calls upon us to begin to think of the entire planet as a kind of community of which we are members.

\subsubsection{Ecological determinants}

- $\quad$ Physiographic (natural forms, forces and process)

Geology

Hydrology

Climate

Biodiversity.

- Topography.

Land configuration: - Land-water outlines, slope.

Natural features: -Land, water, vegetation cover, landscape.

Man-made features -lakes, moats, canals, trafficways, buildings,

- Cultural (social, political and economic factors)

Social influence - Cultural Community resources, Community attitudes and needs, Neighboring uses/historic values.

Political and legal constraints: - political jurisdiction, bylaws zoning, rights of way easements, offside development costs, cost benefit ratio/analysis

Economic factors -land values regional growth potential, taxing structure and assessment, onsite development costs, cost benefit ratio, offsite improvement requirement.

\subsection{Region}

W.I.G. George defines region as "an area whose physical conditions are homogenous" As defined by R. S. Platt (1957), "Region is an area delineated on the basis of general homogeneity of land character and of occupancy." T.J.Woofer who stated, "Region is an area within which the combination of environmental and demographic factors has created one homogeneity of 
economic as social structure", gives a more conceptual definition. French geographer Vidal de la Blache defined the region as "one domain where many dissimilar beings artificially brought together have subsequently adapted themselves to a common existence." However different definitions discussed above, put forward a comprehensive view of the "region as an area, which is homogenous in its own characteristics and distinguished from its surroundings.

All disciplines seem to agree that regions (and hence regionalism) involve diversity at the area level and hence distinctiveness.

\subsection{Ecological region}

The eco-region concept, introduced by Crowley (1967), is based on the notion that such homogeneous ecosystem regions exist in nature and can be delineated and classified. An ecological region is an area with relative homogeneity in ecosystems within which the mosaic of ecosystem components, biotic and abiotic, as well as terrestrial and aquatic, is different than that of adjacent regions (Omernik and Bailey, 1997). Eco-region is any large portion of the Earth's surface over which the ecosystems have characteristics in common.

From above definitions and interface between ecology and architecture Ecological Region can be defined as any portion of the earth having homogeneous characteristics in Architecture, which is shaped by physiographic, topographic and cultural characteristics of the place.

\section{Interface between Architecture and ecology}

Ecological determinants play a crucial role in shaping the built form. Every determinant has a direct relationship with architecture. They all are related to one another and function as an integrated whole seeking symbiotic relationship with architecture. Architecture involves all the living and non living systems in its making and builds a unique ecology of its own. It follows the laws of nature as described by Indian mythology as utpatti - (creation), stithi (Maintenance), Laya (demolition), the cycle of nature. Similarly is the lifecycle of building i.e. prebuilding phase, building phase and post building phase.

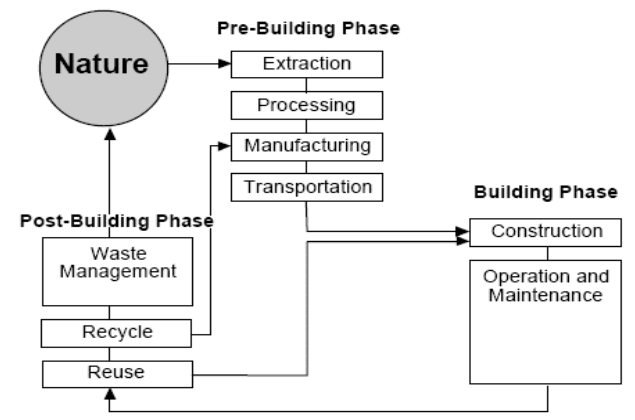

Figure 1: Life cycle of building. 
Scientifically, it obeys the entropy law of Modern Physics According to this law; entropy is the measure of disorder with in a system (in our case buildings). High entropy means that there is a greater disorder and less energy is available for the use where as low entropy means the vice-versa. If contemporary Architecture is analysed in this context, it confines to the high entropy. It is evident that we are facing limitation of available resources and energy. Architecture is the major consumer of them. The ecological equilibrium is lost as a result of built environment.

'Ecology' is the study of living systems and their relations to one another. A living system is an integrated whole which emerge from the relations between its individual parts. Each part reflects the whole but the whole is always different from the mere sum of its parts. Through this basic definition of a living system we can begin to identify the main difference between living and non-living systems. In a non-living system (in our case - buildings) the components together form the whole through a hierarchical structure of construction - each part of the system has its own function and is built specifically to perform this function. The interaction between the components serves the whole but we cannot say that the whole emerges from the interactions between the parts. The study of living systems has influenced architectural design in various ways, although, the results suggest that architects and designers do not truly comprehend how living systems function, but rather try to borrow new ideas from science and ecology and express them in architecture. (As analogies and metaphors).

Building is a management of energy and material. It requires continuous inputs of energy and materials, and gives outputs in the form of waste to its environment. A model which can structure the interactions between a designed system and the ecological environment in terms of these exchanges would therefore be advantageous because such a model would force us, first, to determine the internal activity that takes place within the system, and second, to measure the designed system's dependence upon the ecological environment in terms of the energy and matter that are taken from it and returned to it as a result of the internal activity.

MATERIAL FLOW IN THE BUILDING ECOSYSTEM

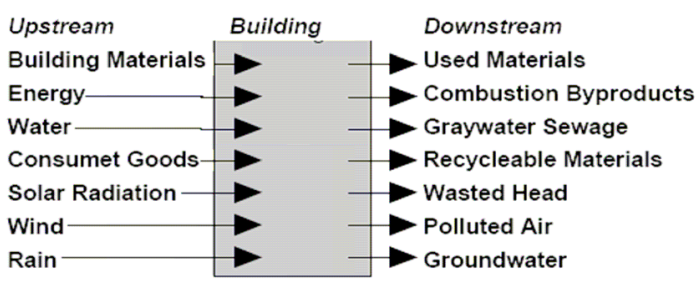

Figure 2: $\quad$ Material flow in the building ecosystem.

Batel Dinur explains three ecological principles, which may change the way in which we perceive and design buildings. The principle of fluctuations 
suggests that buildings may be designed and perceived as places where different cultural and natural processes interact. The building should reflect the processes that occur on site, and the more it allows the processes to be experienced as processes rather than representation of processes, the more it will succeed in connecting people to the reality of the site. The principle of stratification suggests that the building's organization should emerge out of the interactions between its different properties and levels. This kind of organization allows complexity to be managed in a coherent manner. The principle of interdependence suggests that the relations between the building's properties are reciprocal. The 'observer' (designer and user) as well as the site is inherent properties in the building. The interdependence between the properties is ongoing throughout the life of the building.

\subsection{Ecology of architecture}

It is imperative to understand 'The ecology of the building' and its connection to 'The ecosystem of the building'. These two ideas are bridged about context and consequence of systems and flows with emphasis on understanding the concept of the "The building as a snap-shot in a sequence of flows" and the consequences that follow from this. There were a number of immediate system interdependencies in the design of whole buildings. This interdependency meant the inclusion of a variety of designers from several specialties of engineers, electricians, architects, and transportation designers among others. It is important to consider the building from "cradle-to-cradle" given that our frame of reference for a building was often in the form of a static snap-shot from one moment in time. The term "cradle-to-cradle" is used to indicate that components of any building at the end of its useful life could and ought to be recycled into a next structure. Each of the individual components that converge in a building can change over a specific passage of time. Buildings should not be considered static, but should emphasize flexibility and adaptability. This adaptability has a foundation in ecology and nature, but extends to the political landscape, the economy, people in the community, neighbourhoods and social landscape, legal limitations, and other buildings. Once a building is constructed as a complex system; it will be perceived and conceived differently according to its context and to the people that interact with it. A building which will be able to change constantly in relation to natural and cultural processes that interact with it will be a building that is constantly created and re-created not by a single designer but by endless amount of forces and users that come into contact with it.

\section{Regionalism in architecture}

Regionalism is seen as a reaction against the universality and uniformity of contemporary architecture. Architectural regionalism involves cultural, climatic and technological aspects it must also accommodate change. It is a response to a life style, creating places that are particular and distinctive through, a creative synthesis linking past and present and leaving it, own heritage. Regionalism is 
unique response to a particular place and time; it therefore cannot be monolithic. Regionalism is often seen as a foil to International Modernism. Regionalism provides a sense of continuity and tradition that relates to the history and culture of a particular region in contrast to the globalizing forces of International Modernism that are believed to eliminate differences, obliterate individual identities and lead to homogeneous architectural forms.

Alvar Aalto created poetic places out of an intense concern for the needs of what he called 'the little man', and from a deep love of his native landscape. He immersed himself in the particularities of a site, the texture of materials, and the quality of light: wherever architecture is called upon to help define local identities within an increasingly globalised world. Frank Lloyd Wright and Paolo Soleri, who really understood and understand the great region called "Earth," which they celebrated in their work.

Regionalist architecture is not a style; it is a way of life where various facets of that society and solutions to its problems are reflected in the architecture. A direct link with the culture (Culture in architecture results from a group's response to the environment and the fulfilment of its spiritual and physical requirements.) Of the place can be made through the adoption of the traditional responses to climate and creatively using these to solve contemporary problems. (Ken Yeang).

\subsection{Objectives of regionlism}

\subsubsection{To build in harmony with social needs}

Social needs can be achieved through the satisfaction of human emotional demands, and incorporated in to building design. User participation methods are proposed for integrating the local lifestyles into the design e.g. Raj Rewal (India) conceives the idea of modern buildings as an analogue for traditional urban fabrics. He succeeded in translating the broad principles of desert towns like Jasalmer. Here 'Havelis' courtyard houses of complex sections are locked together with streets, squares, gates and doorways in a single system.

\subsubsection{To build in harmony with local resources}

'Local materials' and 'local technology.' Local materials are those indigenous to a specific region and locally available or any material produced locally. Local technology means a combination of traditional craft techniques and modern technology. But in some regions craftsman with traditional skills no longer exist. And local resources are inadequate to respond rapidly to the need for extensive building supplies. Works of Laurie Baker (India) and Hassan Fathy (Egypt) are based on the local resources and technology, which includes innovative, efficient and economical use of available resources, fine craftsmanship.

\subsubsection{To build in harmony with the geographical characteristics of region}

The geographical elements mainly climate and topography. Climate appears to be a rich theme in this. It is a determining factor in architectural regionalism. Climate and topography are the main determinants, which do not change drastically over the years. So these are the key factors in regionalism. e.g. Falling 
water by F.L Wright is example where architecture seeks the dialogue with the nature and integrates harmoniously in to natural landscape.

\subsubsection{To build in harmony with the existing built form}

Its concern is with the physical and aesthetic aspects of building, aiming to retain the specific architectural character of each region by reviving an older, local style. Abstracting elements from the past. It mainly incorporates the abstract qualities of a building, for example massing, solids and voids, proportions, sense of space, use of light and structural principles in their reinterpreted form. It also endeavors to bring back to existence the cultural issues. And then fusing these elements with the contemporary needs of the people and modern technology. But harmony with existing, traditional built forms cannot be achieved on a complete virgin site. Or in an urban area, where all the existing buildings are modern ones?

Works of Charles Correa seems to break the barriers between old and new, monumental and folk. He has tried to work out a viable modern language that draws upon past eras but without mimicking them. He adopted his solutions to the wide range of Indian climate.

It is quite misleading to chunk the whole of modern architecture together as a rootless, functionalist and antisymbiotic phenomenon. The best buildings have been based upon fundamentals extracted from the past; some even provide valuable signposts towards regionalism.

\section{Ecological regionalism}

Interest in ecological regionalism has flowed over the years. Regionalism flourished in Europe during the early nineteenth century and emerged in the U.S. in the form of the western explorations by Lewis and Clark and John Wesley Powell. In the 1930s, Lewis Mumford introduced this term with reference to regional planning. Mumford's regional philosophy aimed at transforming cities into regional centers with diverse ecological and occupational bases (Mumford 1938). This brand of regionalism stood in opposition to the prevailing "metropolitanism" which assumed an inevitable broadening and dominance of cities over outlying regions (Friedmann and Weaver 1979). Metropolitanism overlooked the special biophysical characteristics of a particular region to sustain uniformed growth (Padilla 1996: 102). In contradistinction, Mumford's brand of regional ecology wanted to preserve a balance between rural histories and urban structures. "Garden City" regional planning was the desired method for making it happen.

Ecological regionalism seeks a link with the natural and architectural history of the place. The design should be influenced by the physical and natural characteristic of the place and should reflect and integrate with its natural environment. These include the location, climate, landscape, topography, vegetation and Architecture. It can be defined as the uniqueness of the landscape, architecture of the place. Ecological regionalism refers to a process and an attitude or viewpoint. It is a process wherein responsible consumption of 
resources so as to minimize waste and interact in balanced ways with natural environments and cycles, is practiced. Ecological Regionalism is also a response and an expression of celebration of our existence and respect for nature around us. We cannot live or survive without the use of the resources of the universe and we are filled with awe and respect. A sense of sacredness attaches itself to those resources. Ecological regionalism is about creating an architecture that celebrates and integrates the elements of climate, landscape, and culture that are unique to a place. The use of indigenous building materials would seem to be at the heart of Ecological regionalism. Regionally available building materials and local techniques have contributed extensively in determining architectural forms.

'In a world that is increasingly tending to think alike, it is important to cherish and preserve those elements in our culture and natural history of the place that belong to us and help differentiate us' (Nicholas, 1988).

\section{Conclusion}

Architecture of the $21^{\text {st }}$ Century began as a celebration of the age of industry and technology. Regional variations in architectural styles and building materials enrich the fabric of communities, enhance local economies. .

We should begin our design solutions with the goal of "creating a place" which appropriately connects the project to its location and context. Ecological regionalism reflects the spirit of the Architecture, and a solution rooted in the site. A holistic approach to design requires a proper understanding of the spatial interactions of ecosystems. It is clear that Architecture is shaped by the ecology of the place, if we achieve Ecological regionalism; inevitably regionalism in architecture is achieved. It also helps to conserve natural and regional identity in Architecture. In order to do so, it is useful to consider every built system conceptually as a designed system that has its own life-cycle pattern. Not only with these traditional responsibilities but also with the ecological interactions between the designed system and its environment over its entire physical life cycle.

\section{References}

[1] Yeang Ken, 'Designing with nature-The ecological base for architectural design', McGraw-hill, Inc. 1998

[2] Yeang Ken, 'Tropical urban regionalism- Building in a south east Asian city', A mimar book concept media.

[3] Mcharg Ian, 'Design with Nature', Eugene Feldman, The Falcon press,Philladelphia, U.S.A. 1961

[4] Gadgil M. \& Gupta R, 'Ecology and equity- the use and abuse of nature in contemporary India'.Penguin books in India(p)Ltd, 1995.

[5] Olgyay Victor, 'Design with climate;-A bioclimatic approach to Architectural regionalism', Van Nostrand Reinhold New York, 1992.

[6] Michael Hough, 'City form and natural process,' Routledge London, New York. 
[7] Hyde Richard: 'Climate responsive design', E\& FNspon, London.

[8] Derek Thomas, 'Architecture and the urban environment: A vision for the new age', Architectural Press, Oxford. 2004.

[9] Nicholas C. Markovich, Wolfgang F. E. Preiser \& Fred G. Sturm; 'Pueblo style and regional architecture', VNR; Van Nostrand Reinhold, New York 1990.

[10] Aronin J. E; 'Climate and architecture', Reinhold, 1953.

[11] Jain A.K.; 'Ecology and Natural Resource development Management for sustainable development', Management Publishing Co. 2001.

[12] 'Ecological Climatology:concepts \& application' Cambridge University Press 2002.

[13] Mitsch W \& Sven Erik Jorgensen; 'Ecological Engineering and ecosystem Restoration', 2004.

[14] Gerald M, 'Human Ecology-Basic concepts for sustainable development, Earthscan Publications, U.K.\&U.S.A. 2001

[15] Burgess R, Marisa Carmona and Thed Kolstee; 'The challenge of sustainable cities', Zed books Ltd, London.

[16] Proceedings of the regional seminar in the series exploring Architecture in Islamic cultures, sponsored by The Aga Khan Award for Architecture. 'Architecture and Identity' and 'Regionalism in Architecture'. 